\title{
Natural Frequencies of Vibration of a Magnetoelastic Hollow Cylinder in a Magnetic Field Under Large Deformation
}

\author{
Olokuntoye B. A., Akinola A. P. , Layeni O. P. , Fadodun O. O. \\ Department of Mathematics, Obafemi Awolowo University, Ile-Ife, Nigeria.
}

\begin{abstract}
In this work, the frequency equation of vibration of elastic hollow cylinder in a magnetic field under large deformation is obtained for a semi-linear material. Also, the natural frequencies are numerically calculated and the effect of the magnetic field on the frequency modes are considered. We describe the problem using the equations of elasticity and the Maxwell equations of electromagnetism taking into consideration the effect of the magnetic field on the frequency of vibration of the cylinder. We invoke the appropriate boundary conditions on the Maxwell stress tensor within and on the surface of the cylinder. In the result, the obtained frequency equation showed that it is a generalization of the frequency equation obtained for small deformation theory. The natural frequency of the body increases as the magnetic intensity increases.
\end{abstract}

Keyword-Natural Frequency, Vibration, Semi-linear material, Magnetoelastic cylinder, Large Deformation

\section{INTRODUCTION}

The phenomenon of vibration involves an alternating interchange of potential energy to kinetic energy and vice-versa. Any body having mass and elasticity is capable of oscillatory motion. In engineering, an understanding of the vibratory behavior of mechanical and structural systems is important for the safe design, construction and operation of variety of machines and structures. The failure of most mechanical and structural elements and systems can be associated with vibration. Rumerman and Raynor (1971) considered the natural frequencies of axially symmetric longitudinal vibration of circular cylinders. Laura et al (1974) derived the frequency equation of a cantilever beam that has additional mass attach to it, which is considered as shear force that acted on the free end of the beam. Hutchinson and El-Azhari (1986) developed a series solution of the general threedimensional equations of linear elasticity which was used to find the natural frequencies of the vibration of hollow elastic cylinders with traction free surfaces. Oz and Ozkaya (2005) investigated the natural frequencies of transverse vibration of beam-mass systems for different boundary conditions. Abbas (2006) examined the natural frequencies of vibration of a poroelastic hollow cylinder. Yazdanparast (2011) investigated the vibrations of hollow cylinder in rotation. Abd-Alla (2012) examined the effect of magnetic field and non-homogeneity on the radial vibrations in hollow elastic cylinder under rotation. Yahya and Abd-Alla (2014) considered the radial vibrations of an isotropic elastic rotating hollow cylinder. Using Biot's extension theory, Perati and Gurijala (2015) investigated the torsional vibrations in thick walled hollow poroelastic cylinder. Ebenezer and Ravichandran (2015) considered the free and forced vibrations of hollow elastic cylinders of finite length. Wang et al (2017) examined the frequency equation of flexural vibration of a cantilever beam considering the rotary inertial moment of an attached mass. The objective of this work is to derive the frequency equation of vibration of a magnetoelastic hollow cylinder in a magnetic field under large deformation for a semi-linear material in form of a determinant. Also, the natural frequencies for the modes of a magnetoelastic hollow cylinder were numerically calculated and the effect of the magnetic field on the frequency modes are considered.

\subsection{Geometry of deformation}

\section{PROBLEM SETTING}

Let $\Omega$ be the subset of a three-dimensional Euclidean space $E^{3}$ (i.e $\Omega\left(E^{3}\right.$ ) occupied an isotropic semi-linear elastic body with $r_{1}$ and $r_{2}$ as the inner and outer radii respectively of the hollow cylinder.

We seek for the plane finite deformation of $\Omega$ from an initial configuration of $\Omega_{i}$ into a current configuration of $\Omega_{c}$ by the action, say, of externally applied magnetic field.

The transformation from the initial configuration $\Omega_{i}$ into the current configuration $\Omega_{c}$ is the form

$$
R=R(r, t), \Phi=\theta, Z=z,
$$

Where $(r, \theta, z)$ are the material coordinates in the initial configuration $\Omega_{i}$ and $(R, \phi, Z)$ are the material coordinate in the current configuration $\Omega_{c}$.

The position vectors of every particle in the initial configuration $\Omega_{i}$ and the current configuration $\Omega_{c}$ are respectively given as

$$
\vec{r}=r \overrightarrow{e_{r}}+z \overrightarrow{e_{z}}, \quad \vec{R}=R(r, t) \overrightarrow{e_{R}}+Z \overrightarrow{e_{z}}
$$

where $\overrightarrow{e_{r}}, \overrightarrow{e_{\theta}}, \overrightarrow{e_{z}}$ are the orthogonal local basis vectors associated with the cylindrical coordinates $(r, \theta, z)$ in $\Omega_{i}$ and $\overrightarrow{e_{R}}, \overrightarrow{e_{\phi}}, \overrightarrow{e_{z}}$ are the corresponding local basis vector associated with the cylindrical coordinates $(R, \phi, Z)$ in $\Omega_{c}$. 
Let the geometry of deformation of $\Omega$ from initial configuration $\Omega_{i}$ to current configuration $\Omega_{c}$ be the deformation gradient $\nabla \vec{R}$, where $\nabla$ is the gradient operator in the initial configuration $\Omega_{i}$.

The governing equations of the magneto-elasticity problem we are considering are given as

$$
\left\{\begin{array}{c}
\nabla . \tilde{P}+\tilde{G}=\rho \partial_{t t} \vec{R} \\
\nabla . \vec{E}=4 \pi \rho^{*} \\
\nabla . \vec{B}=0 \\
\nabla \times \vec{E}=-c^{-1} \partial_{t} \vec{B} \\
\nabla \times \vec{H}=4 \pi \vec{\jmath}
\end{array}\right.
$$

where $\vec{E}$ is the electric field intensity vector, $\vec{G}$ is the Lorentz force, $\vec{H}$ is the magnetic intensity, $\vec{B}$ is the magnetic induction vector, $\vec{c}$ is the speed of light, $\vec{J}$ is the current density vector, $\rho$ is the mass density, $\rho^{*}$ is the charge density, and $\tilde{P}$ is the PiolaKirchhoff stress tensor.

The component form of the equation of motion in equation $(3)_{1}$ are

$$
\left\{\begin{array}{c}
\frac{\partial}{\partial r} P_{r r}+\frac{1}{r} \frac{\partial}{\partial \theta} P_{r \theta}+\frac{1}{r}\left(P_{r r}-P_{\theta \theta}\right)+\frac{\partial}{\partial z} P_{r z}+G_{r}=\rho \frac{\partial^{2}}{\partial t^{2}} R(r, t), \\
\frac{\partial}{\partial r} P_{\theta r}+\frac{1}{r} \frac{\partial}{\partial \theta} P_{\theta \theta}+\frac{1}{r}\left(P_{r \theta}-P_{\theta r}\right)+\frac{\partial}{\partial z} P_{r z}+G_{\theta}=0 \\
\frac{\partial}{\partial r} P_{Z r}+\frac{1}{r} \frac{\partial}{\partial \theta} P_{z \theta}+\frac{1}{r} P_{z \theta}+\frac{\partial}{\partial z} P_{z z}+G_{z}=0
\end{array}\right.
$$

where $G_{r}, G_{\theta}$ and $G_{z}$ are components of Lorentz force $\vec{G}$ acting on the body.

\subsection{Energy function and Piolar-kirchhoff stress tensor}

(John, 1960) constructed the energy function for isotropic semi-linear material under large deformation which is given as

$$
\widetilde{W}=\mu I_{1}(\widetilde{U}-\tilde{E})^{2}+\frac{1}{2} \lambda I_{1}^{2}(\widetilde{U}-\tilde{E}),
$$

where $I_{1}(\widetilde{U}-\widetilde{E})$ is the first invariant of the tensor $(\widetilde{U}-\widetilde{E})$ and $\widetilde{U}$ is the right stretch tensor.

We invoke the hypothesis of hyperelasticity and take the Frechet derivative of the energy function (5) with respect to the deformation gradient $\nabla \vec{R}$ to obtain the first Piola-Kirchhoff stress tensor $\widetilde{P}$ which is given as

$$
\begin{gathered}
\tilde{P}(r, \nabla \vec{R})=\frac{\partial}{\partial(\nabla \vec{R})}\left[\mu I_{1}(\widetilde{U}-\tilde{E})^{2}+\frac{1}{2} \lambda I_{1}^{2}(\widetilde{U}-\tilde{E})\right], \\
\tilde{P}(\vec{r}, \nabla \vec{R})=2 \mu \nabla \vec{R}+\left(\lambda I_{1}(\widetilde{U}-\tilde{E})-2 \mu\right) \tilde{O} .
\end{gathered}
$$

The set of equations in (3) above are complemented with constitutive relations given as

$$
\left\{\begin{array}{c}
\vec{J}=\sigma\left(\vec{E}+\frac{1}{c} \frac{\partial}{\partial t} \vec{R} \times \vec{B}\right), \\
\tilde{P}(\vec{r}, \nabla \vec{R})=2 \mu \nabla \vec{R}+\left(\lambda I_{1}(\widetilde{U}-\widetilde{E})-2 \mu\right) \widetilde{O}, \\
\vec{B}=\mu_{e} \vec{H},
\end{array}\right.
$$

where $\tilde{O}$ is the second rank rotation tensor and $\mu_{e}$ is the magnetic permeability of the body.

For a perfect conductor $(\sigma \rightarrow \infty)$, Ohm's law written as equation $(8)_{1}$ becomes

$$
\vec{E}=\frac{1}{c} \frac{\partial}{\partial t} \vec{R} \times \vec{B}
$$

\subsection{Radial vibration in perfect conductor}

For radial vibration, the radial component of the displacement does not vanish identically, i.e

$$
u_{r}=u, u_{z}=u_{\theta}=0
$$

Let the magnetic field $\vec{H}$ be such that

$$
\vec{H}=\overrightarrow{H_{0}}+\vec{h}
$$

where $\overrightarrow{H_{0}}$ (constant) is the externally applied magnetic field acting parallel to the axis of the cylinder and $\vec{h}=\vec{h}(r, t)$ is the perturbation in the magnetic field due to deformation in the electrically conducting cylinder. Substituting equation (9) into equation (3) ${ }_{4}$ of Faraday's law and making use of equation (11) we have,

$$
\vec{h}=-\overrightarrow{H_{0}}+\nabla \times\left(\vec{R} \times \overrightarrow{H_{0}}\right)+\nabla \times \int\left(\frac{\partial}{\partial t} \vec{R} \times \vec{h}\right) d t
$$

In component form, equation (12) can be expressed as

where $h$ satisfies the equation

$$
\vec{h}=(0,0, h) \text {, }
$$

$$
\vec{h}=-H_{0}-\frac{H_{0}}{r} \frac{\partial}{\partial r}(r R(r, t))-\frac{1}{r} \frac{\partial}{\partial r} \int\left(r h \frac{\partial}{\partial t} R(r, t)\right) d t .
$$

The Lorentz force $\vec{G}$ acting on the body is

$$
\vec{G}=\mu_{e} \vec{J} \times \overrightarrow{H_{0}}
$$


substituting the Ampere-Maxwell equation in (3) ${ }_{5}$ into equation (15) and using equation (11) yields

Introducing $\vec{h}=h(r, t) \vec{k}$ in $\nabla \times \vec{h}$ gives

$$
\vec{G}=\frac{\mu_{e}}{4 \pi}(\nabla \times \vec{h}) \times \overrightarrow{H_{0}} .
$$

$$
\nabla \times \vec{h}=\left(0,-\frac{\partial}{\partial r} h(r, t), 0,0\right)=-\frac{\partial}{\partial r} h(r, t) \overrightarrow{e_{\theta}} .
$$

Using equation (17) in equation (16) gives

$$
\vec{G}=\left(-\frac{\mu_{e}}{4 \pi} H_{0} \frac{\partial}{\partial r} h(r, t), 0,0\right),
$$

so that

$$
G_{r}=-\frac{\mu_{e}}{4 \pi} H_{0} \frac{\partial}{\partial r} h(r, t), \quad G_{\theta}=0, \quad G_{z}=0 .
$$

Substituting equation (19) into equation (4), we have

$$
\left\{\begin{array}{c}
\frac{\partial}{\partial r} P_{r r}+\frac{1}{r} \frac{\partial}{\partial \theta} P_{r \theta}+\frac{1}{r}\left(P_{r r}-P_{\theta \theta}\right)+\frac{\partial}{\partial z} P_{r z}-\frac{\mu_{e}}{4 \pi} H_{0} \frac{\partial}{\partial r} h(r, t)=\rho \partial_{t t} \overrightarrow{R,} \\
\frac{\partial}{\partial r} P_{\theta r}+\frac{1}{r} \frac{\partial}{\partial \theta} P_{\theta \theta}+\frac{1}{r}\left(P_{r \theta}-P_{\theta r}\right)+\frac{\partial}{\partial z} P_{\theta z}=0 \\
\frac{\partial}{\partial r} P_{Z r}+\frac{1}{r} \frac{\partial}{\partial \theta} P_{Z \theta}+\frac{1}{r} P_{z \theta}+\frac{\partial}{\partial z} P_{z z}=0
\end{array}\right.
$$

Substituting the components of Piola stress tensor in equation (20), (Olokuntoye et al, 2020) obtained the wave equation in the magnetoelastic semi-linear cylinder as

$$
\begin{gathered}
\frac{(\lambda+2 \mu)}{\rho} \frac{\partial}{\partial r}\left(\frac{1}{r} \frac{\partial}{\partial r}(r R(r, t))\right)-\frac{\mu_{e}}{4 \pi \rho} H_{0} \frac{\partial}{\partial r} h(r, t)=\partial_{t t} \vec{R} \\
h(r, t)=-H_{0}-\frac{H_{0}}{r} \frac{\partial}{\partial r}(r R(r, t))-\frac{1}{r} \frac{\partial}{\partial r} \int\left(r h(r, t) \frac{\partial}{\partial t} R(r, t)\right) d t
\end{gathered}
$$

\section{STRESS FIELDS IN THE CYLINDER}

(Olokuntoye et al., 2020) obtained the solution of equation (21) by taking the first approximation of equation (22) i.e by approximating $h(r, t)$ as $-H_{0}$. The solution was given as

$$
R(r, t)=\left(c_{1} J_{1}(\alpha r)+c_{2} Y_{1}(\alpha r)\right) e^{i \omega t}
$$

\subsection{Zeroth-level stress field in the cylinder}

In order to obtain the stress fields associated with solution (23), we employ the relations

$$
\begin{aligned}
& \frac{d}{d r} J_{1}(\alpha r)=-\alpha J_{2}(\alpha r)+\frac{1}{r} J_{1}(\alpha r), \\
& \frac{d}{d r} Y_{1}(\alpha r)=-\alpha Y_{2}(\alpha r)+\frac{1}{r} Y_{1}(\alpha r) .
\end{aligned}
$$

The non zero components $P_{r r}, P_{\theta \theta}$ and $P_{z z}$ of Piola-Kirchhoff's stress tensor $\tilde{P}$ are

$$
\begin{aligned}
& P_{r r}=(2 \mu+\lambda) \frac{\partial}{\partial r} R(r, t)+\lambda \frac{R(r, t)}{r}-(2 \mu+\lambda), \\
& P_{\theta \theta}=(2 \mu+\lambda) \frac{R(r, t)}{r}+\lambda \frac{R(r, t)}{r}-(2 \mu+\lambda), \\
& P_{z Z}=\lambda\left(\frac{\partial}{\partial r} R(r, t)+\frac{R(r, t)}{r}-2\right) .
\end{aligned}
$$

Substituting the wave solution (23) in equations (26)-(28) and using the relations (24) and (25), we obtain

$$
\begin{aligned}
& P_{r r}=(2 \mu+\lambda)\left[\frac{\partial}{\partial r}\left(c_{1} J_{1}(\alpha r)+c_{2} Y_{1}(\alpha r)\right) e^{i \omega t}-1\right]+\lambda \frac{1}{r}\left(c_{1} J_{1}(\alpha r)+c_{2} Y_{1}(\alpha r)\right) e^{i \omega t}, \\
& P_{r r}=(2 \mu+\lambda)\left[-\alpha\left(c_{2} J_{2}(\alpha r)+c_{2} Y_{2}(\alpha r)\right) e^{i \omega t}-1\right]+2(\mu+\lambda) \frac{1}{r}\left(c_{1} J_{1}(\alpha r)+c_{2} Y_{1}(\alpha r)\right) e^{i \omega t}, \\
& P_{\theta \theta}=(2 \mu+\lambda) \frac{1}{r}\left(c_{1} J_{1}(\alpha r)+c_{2} Y_{1}(\alpha r)\right) e^{i \omega t}+\lambda \frac{\partial}{\partial r}\left(c_{1} J_{1}(\alpha r)+c_{2} Y_{1}(\alpha r)\right) e^{i \omega t}-(2 \mu+\lambda), \\
& P_{\theta \theta}=-\alpha \lambda\left(c_{1} J_{2}(\alpha r)+c_{2} Y_{2}(\alpha r)\right) e^{i \omega t}+2(\mu+\lambda) \frac{1}{r}\left(c_{1} J_{1}(\alpha r)+c_{2} Y_{1}(\alpha r)\right) e^{i \omega t}-(2 \mu+\lambda), \\
& P_{z z}=\lambda\left(\frac{\partial}{\partial r}\left(c_{1} J_{1}(\alpha r)+c_{2} Y_{1}(\alpha r)\right) e^{i \omega t}+\frac{1}{r}\left(c_{1} J_{1}(\alpha r)+c_{2} Y_{1}(\alpha r)\right) e^{i \omega t}-2\right), \\
& P_{z z}=\lambda\left(-\alpha\left(c_{1} J_{2}(\alpha r)+c_{2} Y_{2}(\alpha r)\right) e^{i \omega t}+\frac{2}{r}\left(c_{1} J_{1}(\alpha r)+c_{2} Y_{1}(\alpha r)\right) e^{i \omega t}-2\right) .
\end{aligned}
$$

The current density $\underset{j}{\rightarrow}$ electric field intensity $\underset{E}{\rightarrow}$, and Maxwell's stress $M_{r r}$ generated in the body as a result of $h(r, t) \approx$ $h_{0}(r, t)=-H_{0}$ are

and

$$
\begin{aligned}
& \vec{J}=\frac{1}{4 \pi} \nabla \times \vec{H}(r, t)=-\frac{1}{4 \pi} \nabla \times\left(\overrightarrow{H_{0}}+\vec{h}\right)=\overrightarrow{0}, \\
& \vec{E}=-\frac{1}{c} \frac{\partial \vec{R}}{\partial t} \times \vec{B}=-\frac{\mu_{e}}{c} \frac{\partial \vec{R}}{\partial t} \times\left(\overrightarrow{H_{0}}+\vec{h}\right)=\overrightarrow{0},
\end{aligned}
$$

respectively.

$$
M_{r r}=\frac{\mu_{e}}{4 \pi} H_{0} h=\frac{\mu_{e}}{4 \pi} H_{0}^{2}
$$

\subsection{First-level stress field in the cylinder}


(Olokuntoye et al., 2020) obtained the first-level solution of equation (21) by taking the second approximation of equation (22) i.e by approximating $h(r, t)$ as $-H_{0}-\frac{H_{0}}{r} \frac{\partial}{\partial r}(r R(r, t))$. The solution obtained was given as

$$
\begin{aligned}
& R(r, t)=\left(C_{1} J_{1}(\beta r)+C_{2} Y_{1}(\beta r)\right) e^{i \omega t}, \beta=\frac{\omega}{\psi} \\
& \text { where } \beta=\frac{\omega}{\psi} \text { and } \psi^{2}=\left(\frac{2 \mu+\lambda}{\rho}+\frac{\mu_{e} H_{0}^{2}}{4 \pi \rho}\right),
\end{aligned}
$$

$C_{1}, C_{2}$ are constants and $J_{1}(\beta r), Y_{1}(\beta r)$ are Bessel functions of first and second kinds of order one respectively. Using the relation in equation (24) and (25), the non-zero Piola-Kirchhoff stresses $P_{r r}, P_{\theta \theta}$ and $P_{z z}$ are $P_{r r}=$ $(2 \mu+\lambda)\left[-\beta\left(C_{2} J_{2}(\beta r)+C Y_{2}(\beta r)\right) e^{i \omega t}-1\right]+2(\mu+\lambda) \frac{1}{r}\left(C_{1} J_{1}(\beta r)+C_{2} Y_{1}(\beta r)\right) e^{i \omega t}$,

$P_{\theta \theta}=-\beta \lambda\left(C_{1} J_{2}(\beta r)+C_{2} Y_{2}(\beta r)\right) e^{i \omega t}+2(\mu+\lambda) \frac{1}{r}\left(C_{1} J_{1}(\beta r)+C_{2} Y_{1}(\beta r)\right) e^{i \omega t}-(2 \mu+\lambda)$,

$P_{z z}=\lambda\left(-\beta\left(C_{1} J_{2}(\beta r)+C_{2} Y_{2}(\beta r)\right) e^{i \omega t}+\frac{2}{r}\left(C_{1} J_{1}(\beta r)+C_{2} Y_{1}(\beta r)\right) e^{i \omega t}-2\right)$.

The current density $\vec{\jmath}=(0, j, 0)$ and the electric field $\vec{E}=(0, E, 0)$ generated in the cylinder under consideration are

$$
\begin{aligned}
\vec{J}=\frac{1}{4 \pi} \frac{\partial h}{\partial r} & =-\frac{1}{4 \pi} \frac{\partial}{\partial r}\left(-H_{0}-\frac{H_{0}}{r} \frac{\partial}{\partial r}(r R(r, t))\right), \\
\vec{J} & =\frac{H_{0}}{4 \pi} \frac{\partial}{\partial r}\left(\frac{1}{r} \frac{\partial}{\partial r}(r R(r, t))\right),
\end{aligned}
$$

and

$$
E=\frac{\mu_{e}}{c} \frac{H_{0}}{r} \frac{\partial}{\partial t} R(r, t) \frac{\partial}{\partial r}(r R(r, t))
$$

where $R(r, t)$ is the solution of wave equation $(21)$.

\section{BOUNDARY CONDITIONS AND FREQUENCY EQUATION}

In this section, we are going to obtain the frequency equation for the boundary conditions of an electrically conducting hollow cylinder.

\subsection{Zeroth-level frequency equation}

We proceed to construct the zeroth-level frequency equation which describes various modes of vibration of an electrically conducting semi-linear elastic cylinder. For this, we employ this set of boundary conditions compatible with the problem under consideration:

$$
\begin{gathered}
P_{r r}+M_{r r}+X_{0}=M_{r r}^{*} \text { at } r=a \text { and } r=b, \\
E=E^{*} \text { at } r=a \text { and } r=b,
\end{gathered}
$$

where $X_{0}$ is a given prescribed stress at the boundary of the cylinder, $M_{r r}(r, t)$, is the Maxwell's stress inside the cylinder, $M_{r r}^{*}(r, t)$ is the Maxwell's stress in the vacuum, $E=E(r, t)$ is the electric wave generated in the cylinder, $E^{*}(r, t)$ is the electric wave in the vacuum, and $P_{r r}(r, t)$ is the radial component of the Piola-Kirchhoff stress $\vec{P}$.

We recall that the magnetic wave $\overrightarrow{h^{*}}(r, t)$ and $\overrightarrow{E^{*}}(r, t)$ in vacuum satisfy the electromagnetic field equations

$$
\begin{aligned}
& \nabla^{2} \overrightarrow{h^{*}}=\frac{1}{c} \frac{\partial^{2}}{\partial t^{2}} \overrightarrow{h^{*}}, \\
& \nabla^{2} \overrightarrow{E^{*}}=\frac{1}{c} \frac{\partial^{2}}{\partial t^{2}} \overrightarrow{E^{*}}, \\
& \nabla \times \overrightarrow{h^{*}}=\frac{1}{c} \frac{\partial}{\partial t} \overrightarrow{E^{*}}, \\
& \nabla \times \overrightarrow{E^{*}}=\frac{1}{c} \frac{\partial}{\partial t} \overrightarrow{h^{*}} .
\end{aligned}
$$

The solution of equation (43) is

$$
h^{*}(r, t)=h_{1}^{*}(r) h_{2}^{*}(t)=\left(d_{1} J_{0}\left(\frac{\omega}{c} r\right)+d_{2} Y_{0}\left(\frac{\omega}{c} r\right)\right) e^{i \omega t},
$$

where $d_{1}, d_{2}$ are constants and $J_{0}\left(\frac{\omega}{c} r\right), Y_{0}\left(\frac{\omega}{c} r\right)$ are the Bessel function of the first and second kinds of order zero respectively. Using equation (47) in equation (46) we obtain

$$
E^{*}(r, t)=i \frac{\omega}{c} \frac{\partial}{\partial r}\left(d_{1} J_{0}\left(\frac{\omega}{c} r\right)+d_{2} Y_{0}\left(\frac{\omega}{c} r\right)\right) e^{i \omega t}
$$

For the purpose of the nature of the problem under consideration we set

$$
h^{*}(r, t)=\left\{\begin{array}{c}
d_{1} J_{0}\left(\frac{\omega}{c} r\right) e^{i \omega t}, \text { when } r \leq a \\
d_{2} Y_{0}\left(\frac{\omega}{c} r\right) e^{i \omega t}, \text { when } r \geq b
\end{array}\right.
$$


and

$E^{*}(r, t)=\left\{\begin{array}{l}-i d_{1} J_{1}\left(\frac{\omega}{c} r\right) e^{i \omega t}, \text { when } r \leq a \\ -i d_{2} Y_{1}\left(\frac{\omega}{c} r\right) e^{i \omega t}, \text { when } r \geq b .\end{array}\right.$

The Maxwell's stresses $M_{r r}(r, t)$ and $M_{r r}^{*}(r, t)$ in equation (41) are defined as

$$
\begin{gathered}
M_{r r}(r, t)=-\frac{\mu_{e}}{4 \pi} H_{0} h(r, t), \\
M_{r r}^{*}(r, t)=-\frac{\mu_{e}}{4 \pi \theta} H_{0} h^{*}(r, t),
\end{gathered}
$$

where $h(r, t), h^{*}(r, t)$ are the magnetic waves in the cylindrical body and vacuum respectively.

Introducing equation (49) in equation (52) gives

$$
M_{r r}^{*}(r, t)=\left\{\begin{array}{c}
-\frac{\mu_{e}}{4 \pi} H_{0} d_{1} J_{0}\left(\frac{\omega}{c} r\right) e^{i \omega t}, \text { when } r \leq a \\
-\frac{\mu_{e}}{4 \pi} H_{0} d_{2} Y_{0}\left(\frac{\omega}{c} r\right) e^{i \omega t}, \text { where } r \geq b
\end{array}\right.
$$

Setting $X_{0}$ as

$$
X_{0}=(2 \mu+\lambda)-\frac{\mu_{e}}{4 \pi} H_{0}^{2}
$$

we obtain the following from equations (41) and (42)

$$
\begin{gathered}
X_{11} c_{1}+X_{12} c_{2}=0, X_{21} c_{1}+X_{22} c_{2}=0 \\
d_{1}=d_{2}=0
\end{gathered}
$$

where the coefficient $X_{11}, X_{12}, X_{21}$ and $X_{22}$ in equation (55) are defined as

$$
\begin{aligned}
& X_{11}=-\alpha(2 \mu+\lambda) J_{2}(\alpha a)+\frac{2(\mu+\lambda)}{a} J_{1}(\alpha a), \\
& X_{12}=-\alpha(2 \mu+\lambda) Y_{2}(\alpha a)+\frac{2(\mu+\lambda)}{a} Y_{1}(\alpha a), \\
& X_{21}=-\alpha(2 \mu+\lambda) J_{2}(\alpha b)+\frac{2(\mu+\lambda)}{a} J_{1}(\alpha b),
\end{aligned}
$$

and

$$
X_{22}=-\alpha(2 \mu+\lambda) Y_{2}(\alpha b)+\frac{2(\mu+\lambda)}{a} Y_{1}(\alpha b)
$$

respectively.

For non-zero solution $c_{1}, c_{2}$ in equation (55), we set

$$
\Delta=\left|\begin{array}{ll}
X_{11} & X_{12} \\
X_{21} & X_{22}
\end{array}\right|=0
$$

Equation (57) is the zeroth-level frequency equation of an electrically conducting semi-linear elastic cylinder.

\subsection{First-level frequency equation}

In order to obtain the frequency equation at first-level of approximation, we employ the boundary conditions:

$$
P_{r r}+M_{r r}+X_{0}=M_{r r}^{*} \text {, at } r=a \text { and } r=b
$$


and

$$
h(r, t)+H_{0}=h^{*}(r, t) \text { at } r=a \text { and } r=b .
$$

The solution of the electromagnetic field equations are

$$
h^{*}(r, t)=\left\{\begin{array}{l}
D_{1} J_{0}\left(\frac{\omega}{c} r\right) e^{i \omega t}, \quad \text { when } r \leq a \\
D_{2} Y_{0}\left(\frac{\omega}{C} r\right) e^{i \omega t} \quad \text { when } r \geq b
\end{array}\right.
$$

and

$$
E^{*}(r, t)=\left\{\begin{array}{l}
-i D_{1} J_{1}\left(\frac{\omega}{c} r\right) e^{i \omega t}, \quad \text { when } r \leq a \\
-i D_{2} Y_{1}\left(\frac{\omega}{c} r\right) e^{i \omega t} \quad \text { when } r \geq b
\end{array}\right.
$$

The associated Maxwell's stress in vacuum is

$$
M_{r r}^{*}(r, t)=\left\{\begin{array}{l}
-\frac{\mu_{e}}{4 \pi} H_{0} D_{1} J_{0}\left(\frac{\omega}{c} r\right) e^{i \omega t}, \quad \text { when } r \leq a \\
-\frac{\mu_{e}}{4 \pi} H_{0} D_{2} Y_{0}\left(\frac{\omega}{c} r\right) e^{i \omega t} \quad \text { when } r \geq b
\end{array}\right.
$$

Equations (58) and (59) gives the system of linear equations in variables $C_{1}, C_{2}, D_{1}$ and $D_{2}$

$$
\begin{gathered}
X_{11} C_{1}+X_{12} C_{2}+X_{13} D_{1}+X_{14} D_{2}=0, \\
X_{21} C_{1}+X_{22} C_{2}+X_{23} D_{1}+X_{24} D_{2}=0, \\
X_{31} C_{1}+X_{32} C_{2}+X_{33} D_{1}+X_{34} D_{2}=0, \\
X_{41} C_{1}+X_{42} C_{2}+X_{43} D_{1}+X_{44} D_{2}=0 .
\end{gathered}
$$

The coefficient of $X_{11}, X_{12}, \ldots, X_{44}$, in the above equation (63)-(66) are written as

$$
\begin{gathered}
X_{11}=-\beta(2 \mu+\lambda) J_{2}(\beta a)+\frac{2(\mu+\lambda)}{a} J_{1}(\beta a)+\frac{\mu_{e}}{4 \pi} H_{0}^{2}\left(\frac{2}{a} J_{1}(\beta a)-\beta J_{2}(\beta a)\right) \\
X_{12}=-\beta(2 \mu+\lambda) Y_{2}(\beta a)+\frac{2(\mu+\lambda)}{a} Y_{1}(\beta a)+\frac{\mu_{e}}{4 \pi} H_{0}^{2}\left(\frac{2}{a} Y_{1}(\beta a)-\beta Y_{2}(\beta a)\right) \\
X_{13}=\frac{\mu_{e}}{4 \pi} H_{0} J_{0}\left(\frac{\omega}{c} a\right), X_{14}=0, \quad X_{34}=0, \quad X_{44}=J_{0}\left(\frac{\omega}{c} b\right) \\
X_{21}=-\beta(2 \mu+\lambda) J_{2}(\beta b)+\frac{2(\mu+\lambda)}{a} J_{1}(\beta b)+\frac{\mu_{e}}{4 \pi} H_{0}^{2}\left(\frac{2}{a} J_{1}(\beta b)-\beta J_{2}(\beta b)\right) \\
X_{22}=-\beta(2 \mu+\lambda) Y_{2}(\beta b)+\frac{2(\mu+\lambda)}{a} Y_{1}(\beta b)+\frac{\mu_{e}}{4 \pi} H_{0}^{2}\left(\frac{2}{a} Y_{1}(\beta b)-\beta Y_{2}(\beta b)\right)
\end{gathered}
$$




$$
\begin{aligned}
& X_{24}=\frac{\mu_{e}}{4 \pi} H_{0} J_{0}\left(\frac{\omega}{c} a\right), X_{23}=0, \quad X_{43}=0, \quad X_{33}=J_{0}\left(\frac{\omega}{c} a\right) \\
& X_{31}=H_{0}\left(\frac{2}{a} J_{1}(\beta a)-\beta J_{2}(\beta a)\right), \quad X_{32}=H_{0}\left(\frac{2}{a} Y_{1}(\beta a)-\beta Y_{2}(\beta a)\right) \\
& X_{41}=H_{0}\left(\frac{2}{b} J_{1}(\beta b)-\beta J_{2}(\beta b)\right), \quad X_{42}=H_{0}\left(\frac{2}{b} Y_{1}(\beta b)-\beta Y_{2}(\beta b)\right)
\end{aligned}
$$

For non-zero solution $C_{1}, C_{2}, D_{1}$, and $D_{2}$

$$
\Delta=\left|\begin{array}{cccc}
X_{11} & X_{12} & X_{13} & 0 \\
X_{21} & X_{22} & 0 & X_{24} \\
X_{31} & X_{32} & X_{33} & 0 \\
X_{41} & X_{42} & 0 & X_{44}
\end{array}\right|=0
$$

Equation (67) is the first level frequency equation for a perfectly conducting semi-linear elastic hollow cylinder under consideration.

\section{NUMERICAL RESULTS}

\begin{tabular}{|c|c|c|}
\hline$\zeta$ & $H_{0}=10^{5}$ & $H_{0}=10^{10}$ \\
\hline 0.1 & 4.600489912 & 4.741371586 \\
\hline 0.2 & 5.911873584 & 6.092304748 \\
\hline 0.3 & 7.597071168 & 7.828151931 \\
\hline 0.4 & 9.762639461 & 10.05858459 \\
\hline 0.5 & 12.5455096 & 12.92452227 \\
\hline 0.6 & 16.12164534 & 16.60703595 \\
\hline 0.7 & 20.71716949 & 21.33878818 \\
\hline 0.8 & 26.6226618 & 27.41873278 \\
\hline 0.9 & 34.21153269 & 44.77870011 \\
\hline
\end{tabular}

In this section, we evaluated the natural frequencies for the first four modes of an elastic hollow cylinder of various thickness for two different values of externally applied magnetic field. The results are shown in the tables below

Table 1: First mode of Natural Frequencies of an magnetoelastic hollow cylinder of various thickness (५) for two different values of externally applied magnetic

Table 2: Second mode of Natural Frequencies of magnetoelastic hollow cylinder of various thickness $(\zeta)$ for two different values of externally applied magnetic

\begin{tabular}{lcl}
\multicolumn{1}{c}{ field } & $\boldsymbol{H}_{\mathbf{0}}=\mathbf{1 0}^{\mathbf{1 0}}$ \\
\hline $\mathbf{0 . 1}$ & $\boldsymbol{H}_{\mathbf{0}}=\mathbf{1 0}^{\mathbf{5}}$ & 12.7371271 \\
\hline $\mathbf{0 . 2}$ & 12.20800396 & 16.52081535 \\
\hline $\mathbf{0 . 3}$ & 15.68793269 & 21.42848523 \\
\hline $\mathbf{0 . 4}$ & 20.15982573 & 27.79402647 \\
\hline $\mathbf{0 . 5}$ & 25.9064455 & 36.05051403 \\
\hline $\mathbf{0 . 6}$ & 33.29115675 & 46.75967202 \\
\hline $\mathbf{0 . 7}$ & 42.78090245 & 60.65009019 \\
\hline $\mathbf{0 . 8}$ & 54.97572909 & 78.66679301 \\
\hline
\end{tabular}

Table 3: Third mode of Natural Frequencies of magnetoelastic hollow cylinder of various thickness $(\zeta)$ for two different values of externally applied magnetic field 


\begin{tabular}{lcc}
\hline$\zeta$ & $\boldsymbol{H}_{\mathbf{0}}=\mathbf{1 0}^{\mathbf{5}}$ & $\boldsymbol{H}_{\mathbf{0}}=\mathbf{1 0}^{\mathbf{1 0}}$ \\
\hline $\mathbf{0 . 1}$ & 20.43234346 & 20.80118 \\
\hline $\mathbf{0 . 2}$ & 25.43096458 & 27.3854 \\
\hline $\mathbf{0 . 3}$ & 32.68013854 & 36.05372 \\
\hline $\mathbf{0 . 4}$ & 41.99571165 & 47.46582 \\
\hline $\mathbf{0 . 5}$ & 53.96671726 & 62.49021 \\
\hline $\mathbf{0 . 6}$ & 69.3500945 & 82.27028 \\
\hline $\mathbf{0 . 7}$ & 89.11855032 & 108.3113 \\
\hline $\mathbf{0 . 8}$ & 114.5220647 & 142.5952 \\
\hline $\mathbf{0 . 9}$ & 185.3921045 & 199.6127 \\
\hline
\end{tabular}

Table 4: Fourth mode of Natural Frequencies of magnetoelastic hollow cylinder of various thickness $(\zeta)$ for two different values of externally applied magnetic

\begin{tabular}{lcc} 
& \multicolumn{1}{c}{ field } & $\boldsymbol{H}_{\mathbf{0}}=\mathbf{1 0}^{\mathbf{1 0}}$ \\
\hline $\boldsymbol{\zeta}$ & $\boldsymbol{H}_{\mathbf{0}}=\mathbf{1 0}^{\mathbf{5}}$ & 27.930159 \\
\hline $\mathbf{0 . 1}$ & 27.3691174 & 36.62412121 \\
\hline $\mathbf{0 . 2}$ & 35.28349447 & 48.02429711 \\
\hline $\mathbf{0 . 3}$ & 45.48648622 & 62.97306358 \\
\hline $\mathbf{0 . 4}$ & 58.63989551 & 82.57500838 \\
\hline $\mathbf{0 . 5}$ & 75.5968999 & 108.2785499 \\
\hline $\mathbf{0 . 6}$ & 97.45739184 & 141.9829632 \\
\hline $\mathbf{0 . 8}$ & 125.6393217 & 186.17872 \\
\hline $\mathbf{0 . 9}$ & 161.970671 & 244.1315142 \\
\hline
\end{tabular}

\section{CONCLUSIONS}

The frequency equation of vibration of a magnetoelastic hollow cylinder in a magnetic field under large deformation for a semilinear material was obtained. Also, the natural frequencies for the first four modes of a magnetoelastic hollow cylinder of various thickness for two different values of externally applied magnetic field were numerically calculated. It is shown in the tables above that the natural frequencies of the magnetoelastic hollow cylinder increases as the thickness of the hollow cylinder increases. Furthermore, the effect of the magnetic field on the frequency modes were considered. It is clearly shown from the tables above that the natural frequencies of the magnetoelastic hollow cylinder increases as the externally applied magnetic field intensity increases. The results shows that the natural frequencies obtained are greater than the corresponding small deformation case.

\section{REFERENCES}

[1] Abbas, I. A. Natural frequencies of a poroelastic hollow cylinder. Acta Mechanica, 2006; 186(1):229-237.

[2] Abd-Alla, A. M., G. A. Yahya, and S. R. Mahmoud. "Effect of magnetic field and non-homogeneity on the radial vibrations in hollow rotating elastic cylinder." Meccanica 2013; 48(3):555-566.

[3] Ebenezer, D.D., Ravichandran, K. and Padmanabhan, C. Free and forced vibrations of hollow elastic cylinders of finite length. The Journal of the Acoustical Society of America, 2015; 137(5): 2927-2938.

[4] Laura, P.A.A., Pombo, J.L. and Susemihl, E.A. A note on the vibrations of a clamped-free beam with a mass at the free end. Journal of Sound and Vibration, 1974; 37(2):161-168.

[5] Öz, H.R. and Özkaya, E. Natural frequencies of beam-mass systems in transverse motion for different end conditions. Mathematical and Computational Applications, 2005; 10(3):369-376.

[6] Olokuntoye B.A., Akinola A.P., Layeni O.P. and Fadodun O.O., Radial vibration of a magnetoelastic hollow cylinder under finite deformation. Solid State Technology, 2020; In print.

[7] Paul, H.S. and Venkatesan, M. Vibrations of a hollow circular cylinder of piezoelectric ceramics. The Journal of the Acoustical Society of America, 1987; 82(3):952-956.

[8] Perati, M.R. and Gurijala, R. Investigation of torsional vibrations in thick walled hollow poroelastic cylinder using Biot's extension theory. Sadhana, 2015;40(6):1925-1935.

[9] Rumerman, M. and Raynor, S. Natural frequencies of finite circular cylinders in axially symmetric longitudinal vibration. Journal of Sound and Vibration, 1971;15(4):529-543.

[10] Wang, B., Wang, Z. and Zuo, X. Frequency equation of flexural vibrating cantilever beam considering the rotary inertial moment of an attached mass. Mathematical Problems in Engineering, 2017; 4:1-5

[11] Yahya, G.A. and Abd-Alla, A.M. Radial vibrations in an isotropic elastic hollow cylinder with rotation. Journal of Vibration and Control, 2016; 22(13):3123-3131.

[12] Yazdanparast, L., Mohammadi, A.K. and Abdi, B. The Vibrations of rotating hollow cylinder. International review of mechanical engineering, 2011;5(3):409-421. 